\title{
Bioetica della strumentazione medica: il rene artificiale
}

\author{
Mario Timio $^{1,2}$ \\ ${ }^{1}$ Dipartimento di Medicina Interna e Dialisi, Ospedale Foligno, Foligno (PG) \\ ${ }^{2}$ Presidente Scientifico Centro di Bioetica della Regione Umbria, Perugia
}

THE BIOETHICAL IMPLICATIONS OF MEDICAL INSTRUMENTATION: ARTIFICIAL KIDNEY

Abstract. A technology of medicine arose in the nineteenth century with the direct application of devices and instruments to a part of the body for diagnosis and treatment. The technological innovations are a part of scientific, economic and sociological dimensions of medicine, and while easily delineated as a narrow theme in itself, it involves the broad field of bioethics. The instrumentation is neutral toward man's health, but when he falls in a disease and has a vast collection of emotional needs, he wants reassurance, he wants to be listened, he wants to feel that it makes difference to the physician whether he lives or dies. He wants to feel that he is in physician's thoughts and not only in the mechanisms of diagnosis or treatment instruments. This is the concern of modern bioethics which tries to bring the instrument closer and closer to the sick person. The artificial kidney as a specific and unique machine to treat end-stage renal disease complicates the bioethics approach to the dialysis patient because of the economical and political interferences gathered under the fourth factor of the Hippocrates' triangle.

Key words: Medical instrumentation, Bioethics, Hemodialysis

Conflict of interest: None.

Financial support: None.

Accettato: 29 Maggio 2014

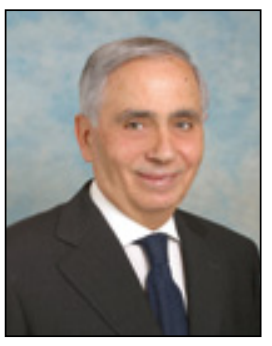

Mario Timio

\section{La strumentazione come elemento cardine nella diagnostica e nella terapia}

"La medicina è la sola professione che lotta incessantemente per distruggere la ragione della propria esistenza". In queste parole di James Bryce c'è l'essenza della pratica medica. Ma anche il suo contrario. Il medico lotta per distruggere la malattia, ma un malato gli sta sempre di fronte. La malattia è il lato notturno della vita che provoca lo spasmodico desiderio del giorno. Con l'aiuto del medico. Non è condivisibile neanche il pensiero del poeta Ezra Pound, quando asserisce che "può darsi che tutte le scienze comincino con la sofferenza e con la pietà. Ma una volta che hanno preso l'avvio, non c'è più posto per le emozioni". In medicina non è proprio così. Le emozioni in medicina esistono, anche se prendono vari nomi e colorite connotazioni. Le emozioni si caricano di significati e di scelte che conducono alla diagnosi e alla cura della persona malata. Diagnosi e cura, a loro volta, inseriti in percorsi metodologici, clinici e strumentali che connotano il tessuto di ogni malattia. In questo tessuto fin da Ippocrate si sono inseriti germi di etica clinica. E, nell'etica clinica, entra sempre più prepotentemente la strumentazione medica.

Come si embricano la strumentazione medica e la biotecnologia nel contesto della tematica della bioetica clinica e segnatamente negli interrogativi morali sollevati durante il processo di diagnosi e cura? Dal momento che la clinica e la bioetica devono prendere decisioni particolari basate su una metodologia che coinvolge l'uso di strumenti diagnostici e curativi, si evince che la bioetica e la strumentazione percorrono un tratto comune e necessario per il rapporto medico-malato. Se è vero che la strumentazione applicata alla medicina ha consentito progressi straordinari nella diagnostica e nella terapia, tuttavia i costi legati a tali progressi sono stati e sono molto elevati. Costi non solo diretti del procedimento diagnostico e curativo, ma anche costi indiretti connessi a interventi che curano senza guarire, per esempio la dialisi. Il conseguente aumento di pazienti cronici pone interrogativi etici importanti e crea prospettive di sostenibilità. Quindi, 1'incontro medico-assistito, che deve tenere conto anche dell'appropriatezza della collocazione strumentale e tecnologica, si caratterizza per una sua fondamentale componente etica. 


\section{Asimmetria tra conoscenza scientifica e prescrizione di bioetica clinica}

All'inizio dell'era dialitica, i problemi bioetici erano molto pregnanti in termini di selezione, accettazione, tempistica, interruzione e costo. Di problemi non solo clinici doveva, quindi, tenere conto il medico in procinto di iniziare la dialisi. Ciò è inseribile nel contesto del coinvolgimento umanistico della evoluzione della scienza. Un breve excursus storico ci indica che tale coinvolgimento è emerso solo dopo la rivoluzione scientifica, cioè nel periodo compreso tra il 1543, con la pubblicazione del De Revolutionibus di Niccolò Copernico, e il 1687, anno di stampa del Philosophiae Naturalis Principia Mathematica di Isaac Newton (1). Proprio durante tale periodo si dà avvio all'era strumentale; la medicina fa un salto di qualità mediante l'introduzione dello strumento in fase diagnostica e terapeutica. È un approccio nuovo alla realtà biologica in virtù della diffusione del concetto di misura direttamente connesso a quello di "macchina". Questa offre una forma nuova di sapere che investe tutto lo scibile umano. In tale rivoluzione scientifica lo strumento diventa parte integrante del sapere scientifico. "Lo strumento è dentro la teoria; diventa teoria esso stesso" (2). Sulla scia dell'"esplosione" strumentale si legalizza l'uso dell'apparecchio nell'acquisizione della conoscenza e della manipolazione della realtà biologica. Il percorso che conduce a tale legalizzazione attraversa la considerazione dello strumento come aiuto, se non come sostituzione, dei sensi. Ciò vale sia per l'apparecchio diagnostico che per quello curativo. Hooke, storico della medicina, è dell'avviso che "la prima cosa da fare riguardo ai sensi è un tentativo di supplire alla loro infermità con strumenti e cioè aggiungere organi artificiali a quelli naturali"(3). Così, abbiamo il microscopio e il telescopio amplificatori rispettivamente dell'infinitamente piccolo e dell'infinitamente lontano, che suppliscono all'occhio dell'uomo, incapace di vedere "naturalmente" realtà non alla portata della sua vista. Ma ciò è ancora normale per la fisiologia dell'uomo. Lo strumento applicato in medicina può dare di più: supplire alla funzione di organi malati, sostituendoli. Così, abbiamo la protesi dentaria e acustica, l'arto artificiale, il rene e il cuore artificiali e altri organi non funzionanti, tranne il cervello (finora). Nell'ampliamento dei sensi normali o nella sostituzione degli organi malati, l'approccio diagnostico e curativo si configura come un procedimento basato più su indici strumentali o laboratoristici che sul contatto diretto sensoriale con il paziente. Nella diagnostica, la ricerca di elementi rilevabili senza speciali componenti sensoriali, ma affidati a dati strumentali rivela, tra l'altro, la tendenza della moderna medicina a sostituire l'occhio clinico, cioè un senso, con l'occhio strumentale. Anche nell'iter seguito dalla strumentazione curativa o sostitutiva si intravede la tendenza ad applicare la tecnica alla malattia, sganciata dal malato con le sue esigenze, le sue aspettative e le sue paure. In questo contesto, lo strumento deforma il rapporto di fiducia medico-assistito, nel quale svetta la "figura" dell'apparecchio per eccellenza: il computer. Come argomenta Lowi della Cornell University (USA) al malato-persona si presentano due possibilità: la totale indifferenza o la passiva accettazione del sistema in cui il dittatore è il computer. Ciò riporta alla fase pre-fisica della medicina: l'atto medico si articola in un processo intellettuale basato su teorie mediche e su assunti dogmatici che prevedono uno scarso contatto fisico con il paziente.

\section{Problemi bioetici nel ritorno alla fase pre-fisica della medicina}

In questa fase è carente la componente strumentale diagnostica, essendo invece quella curativa molto esuberante. Successivamente, nella fase fisica e sensoriale, l'esame clinico inizia ad avvalersi di strumenti diagnostici naturali: l'occhio per osservare, la mano per palpare, il naso per fiutare e l'orecchio per ascoltare. Si realizza quanto ipotizzato da Ippocrate circa l'esame clinico, che deve tenere in considerazione "ciò che è possibile vedere, toccare $\mathrm{e}$ intendere e ciò che è afferrabile attraverso la vista, il tatto, l'udito, la parola e il pensiero". I medici romani sono grandi fautori della strumentazione diagnostica sensoriale; di contro, tengono in scarsa considerazione la strumentazione curativa (chirurgica). I seguaci della scuola di Salerno esaltano invece la strumentazione curativa in cui si intravede l'inizio di una pre-etica clinica, tenendo in grande considerazione l'infermo nell'ambito di un esaltato rapporto medico-malato. Ancora più attenta al malato e all'etica professionale è la scuola di Montpellier che, con le parole di Guy de Chauliac, sintetizza il suo orientamento accademico: "il chirurgo sia prezioso per il malato, benevolo nei confronti dei suoi compagni e saggio nella sua prognosi; sia pietoso e misericordioso, non estorca argento, ma riceva salario secondo il suo lavoro, le possibilità del malato, la qualità del risultato e la sua dignità"(4).

Il passaggio alla terza fase storica dell'approccio medico, quella strumentale, riconduce il rapporto curante-paziente all'era pre-fisica poiché si configura il distacco del medico dalla persona malata. Lo strumento, nato da un problema patologico, contribuisce ad ampliare le conoscenze delle branche che lo hanno sollecitato e a modificare la natura stessa del fenomeno che studia. Tuttavia, paradossalmente, più lo strumento si avvicina alla realtà biologica, più allontana l'uomo dall'uomo. E in dialisi cosa avviene? Come può la bioetica interferire con il trattamento sostitutivo o accompagnarlo? È indubbio che la dialisi, come il trapianto renale, imponga obblighi etici al medico come in poche altre condizioni terapeutiche. Lo abbiamo accennato: scelta, selezione, tempo, durata, luogo, interruzione, costi, comorbidità. Ogni componente solleva una miriade di interrogativi ai quali il medico deve dare risposte. In sintesi, se la dialisi ha prolungato e prolunga la vita a molti malati, ha creato considerevoli difficoltà collaterali non solo agli stessi pazienti, ma ai familiari, ai caregiver e al personale sanitario. Sovente gli infermieri in dialisi soffrono di sindrome di burn-out per essere il ricettacolo diretto di tutte le giustificate lamentele dei pazienti. E il medico?

\section{L'emodialisi entra nel quarto fattore M: la rilevanza bioetica}

Nell'antichità la sanità era inserita nel cosiddetto triangolo di Ippocrate o delle tre M (una per angolo): Medico, Malato, 
Malattia. Nell'era moderna il triangolo si è trasformato in rettangolo, con il quarto angolo occupato da un'altra M, Mondo esterno, costituito dalla tecnologia diagnostica e curativa, dall'epidemiologia, dalla prevenzione, dalla politica e dall'economia sanitaria e dall'organizzazione dei servizi. L'aggiunta di un quarto angolo comporta stravolgimenti epocali in campo medico. E, soprattutto, pone tra il curante e il curato muri spesso insormontabili. Non è, quindi, solo lo strumento rappresentato dal rene artificiale a creare problemi di interattività, ma è lo strumento inserito nel contesto della quarta $\mathrm{M}$ a rendere difficile il rapporto medico-malato e a spingere la bioetica clinica nella fase della rilevanza e del recupero di competenze antropologiche (5).

Elvisio era un ragazzo emodializzato nel reparto da me diretto. Tanti problemi clinici, ma risolvibili o, almeno, ridimensionabili con le terapie disponibili. Ma non era la clinica a preoccupare Elvisio. A causa dell'incremento dei dializzati, il centro inizia a soffrire di disservizi. Si allerta ripetutamente la Direzione della ASL, che rimane sempre sorda. A questa sordità Elvisio risponde con un'iniziativa scioccante e originale: sciopero della dialisi. Salta un turno, ne salta due, ne salta tre. E irremovibile a tutte le sollecitazioni. Compresa la mia. Vuole che il presidente della ASL gli dia garanzie scritte sull'immediata esecutività delle migliorie logistiche del centro dialisi. Ottiene lo scopo e riprende l'emodialisi. Quella di Elvisio è un'emblematica vicenda, con filamenti di altruismo, inseribile nell'angolo $\mathrm{M}$ del rettangolo sanitario. Esiste il Medico, è presente la Malattia, si preoccupa il Malato. Ma, tra il medico e il malato, si inseriscono non solo lo strumento, ma anche la politica sanitaria connessa in qualche modo alla terapia strumentale effettuata con il rene artificiale. Appunto il quarto fattore $\mathrm{M}$, che, in questo caso, complica anche l'approccio bioetico. Elvisio in sciopero è una persona con una propria dignità che le istituzioni sembrano calpestare, ma che il medico deve salvaguardare, infondendo fiducia, assicurando vicinanza e aiutando a ritrovare il valore della propria esistenza, che, in uno slancio di altruismo e di solidarietà, stava per perdere. La dialisi più di altre condizioni curative determina rilevanti trasformazioni non solo organiche, ma di tutta la sfera della vita della persona. Vengono coinvolti l'essere fisico, l'essere relazionale, l'essere emozionale e l'essere spirituale. Viene sollecitato anche un sano egotismo, che è la spinta necessaria di adattamento e di sopravvivenza. Elvisio ha invece innestato la marcia dell'altruismo e della solidarietà. Comunque, tutti sentimenti che richiedono alti dosi di bioetica per indirizzarli verso traguardi sostenibili (6).

\section{Conclusioni}

L'apparecchio nell'era strumentale, mentre rimuove notevoli difficoltà diagnostiche e curative, riporta all'era pre-fisica della storia della medicina per quanto concerne il rapporto medico-malato: esistono la malattia da una parte e il malato dall'altra. Ciò configura emergenti problemi di bioetica che rimangono gli unici a legare curante e curato. Nell'era moderna o strumentale a organizzare, ma, talvolta, a complicare, l'iter della medicina diagnostica e, ancora di più, curativa, come la dialisi, è emerso il fattore $\mathrm{M}$, aggiunto a quelli che costitui- scono il triangolo di Ippocrate (Medico, Malattia, Malato) e che hanno indirizzato la sanità per molti secoli. Nel fattore M si affollano, oltre alla tecnologia, la medicina preventiva, l'epidemiologia, la politica e l'economia sanitarie e l'organizzazione dei servizi, tanto per citare qualche esempio. Ognuno di essi richiede alti dosi di bioetica per rendere la medicina all'altezza della dignità del medico e del malato di ippocratica memoria (7). È l'insieme dei fattori inseriti nel fattore $\mathrm{M}$, che, posizionandosi tra il paziente e il dializzatore, crea un complesso di problematiche di etica clinica al quale il medico è chiamato a fornire risposte tempestive e circostanziate, pur essendo il campo della strumentazione nefrologica ristretto.

\section{Riassunto}

Dal momento che la tecnica dialitica è oggi altamente perfezionata e sofisticata, rimangono due punti essenziali: a) la sopravvivenza non è più abbastanza e b) i problemi bioetici innestati a ventaglio richiedono risposte circostanziate adattate al singolo dializzato. È questa una nuova sfida al nefrologo del XXI secolo. Nell'era pre-fisica della medicina non esistevano categorie bioetiche, anche se da una parte si poneva il Medico con le sue competenze e, dall'altra, la Malattia, ma il Malato (terza componente o terza $M$ del triangolo di Ippocrate) era escluso da ogni decisione. Nell'era fisica che si caratterizza per l'uso degli strumenti sensoriali (vista, tatto, udito e fiuto), il medico è fisicamente vicino al malato ed è abbozzata una sorta di pre-etica clinica. Nell'era attuale o strumentale, l'apparecchio diagnostico o curativo si inserisce tra il Medico e il Malato, che rimane estraneo al processo del recupero della salute. Per questo sono richieste alte dosi di bioetica, volte a preservare la dignità del paziente, a garanzia del rispetto, del suo best interest e del suo bene. A rendere più cogente l'impegno del nefrologo è l'alleanza con il paziente per superare quello che è definito il fattore $\mathrm{M}$ (o quarta $\mathrm{M}$, per distinguerla dalle tre classiche del triangolo di Ippocrate), nel quale convergono, oltre alle problematiche strumentali del dializzatore, le nuove componenti del sapere medico, come la politica e l'economia sanitarie, l'organizzazione dei servizi, l'epidemiologia e la medicina difensiva.

Parole chiave: Strumentazione medica, Bioetica, Emodialisi

Dichiarazione di conflitto di interessi: L'Autore dichiara di non avere conflitto di interessi.

Contributi economici agli Autori: L'Autore dichiara di non aver ricevuto sponsorizzazioni economiche per la preparazione dell'articolo.

Indirizzo dell'Autore:

Prof. Mario Timio

Via XX Settembre 22

06121 Perugia

timma@libero.it 


\section{Bibliografia}

1. D'Agostino S. Storia della strumentazione scientifica e sviluppo della scienza. Epistemologia, Metodologia, Clinica e Storia della Scienza. Cossidente Editore, Roma 1978: 9-15.

2. Reale G, Antiseri D. Il pensiero occidentale dalle origini a oggi. Vol. II, Editrice La Scuola, Brescia 1983: 147.

3. Hooke R in Keele KD. The evolution of Clinical Medicine, Charles C. Thomas, Sprinfield, Ill. 1936: 16.
4. de Chauliac G. Chirurgia Magna, in: Timio M. Bioetica della strumentazione nel rapporto medico-malato. Seminario di Bioetica, Aracne Editrice, Roma 2008: 100.

5. Timio M. Bioetica della strumentazione nel rapporto medicomalato. Seminario di Bioetica, Aracne Editrice, Roma 2008: 102-3.

6. Veatch RM. Case studies in medical Ethics. Harvard University Press, Cambridge 1977: 168.

7. Chopra D. Creating health, Thorsons, London 1985: 151. 